\title{
Primate Speciation Links to Massive and Directional Shrinkage of the Dinucleotide Short Tandem Repeat Compartment.
}

\section{Masoud Arabfard}

Baqiyatallah University of Medical Sciences

Mahmood Salesi

Baqiyatallah University of Medical Sciences

Iman Arabipour

Islamic Azad University, Tehran

Reza Najafipour

Qazvin University of Medical Sciences

\section{Ahmad Delbari}

University of Social Welfare and Rehabilitation Sciences

Hamid Reza Khorram Khorshid

Hope Generation Foundation, Iran

Mina Ohadi ( $\nabla$ ohadi.mina@yahoo.com )

University of Social Welfare and Rehabilitation Sciences

\section{Research Article}

Keywords: dinucleotide, short tandem repeat, directional, primate, mouse, speciation

Posted Date: January 29th, 2021

DOl: https://doi.org/10.21203/rs.3.rs-114156/v1

License: (9) This work is licensed under a Creative Commons Attribution 4.0 International License. Read Full License 


\section{Abstract}

The evolutionary trend of short tandem repeats (STRs) at the crossroads of speciation remains largely elusive and attributed to random evolution for the most part. Here we investigated the dinucleotide STR compartment in primate speciation. We selected six species, which shared sequential chronological ancestors, including mouse, macaque, gorilla, chimpanzee, bonobo, and human, and collected three sets of data on the abundance of all classes of dinucleotide STRs ( $\geq 6$-repeats) for three regions of every chromosome, each region spanning $10 \mathrm{Mb}$ of DNA. In all three datasets, we found consistent directional shrinkage of the dinucleotide STR compartment in all the primate species selected vs. mouse, as follows: mouse>macaque>great apes. The $>20$-repeat STRs were the most significantly affected as a result of this shrinkage. We propose that massive and directional shrinkage of the dinucleotide STR compartment had a decisive link with primate speciation. This is a prime instane of massive directional STR trend in multiple speciation.

\section{Introduction}

With an exceptionally high degree of polymorphism and plasticity, short tandem repeats (STRs) are a spectacular source of variation required for speciation and evolution ${ }^{1-3}$. The impact of STRs on speciation is supported by their various functional implications, such as gene expression, alternative splicing, and translation ${ }^{3-10}$.

While a limited number of studies indicate that purifying selection and drift can shape the structure of STRs at the inter- and intra-species levels ${ }^{11-16}$, the global trend of STR evolution at the crossroads of primate speciation remains largely unknown.

The most common STRs in the human genome are dinucleotide repeats ${ }^{17}$. Here we analyzed the evolutionary trend of this category of STRs in six selected species encompassing rodent, Old World monkey, and great apes.

\section{Materials And Methods}

\section{Extraction of STRs from genomic se quences.}

The abundance of dinucleotide STRs was studied in six selected species, which shared sequential chronological ancestors, including mouse, macaque, gorilla, chimpanzee, bonobo, and human. We designed a software package in C\# environment (Suppl. 1) (https://github.com/arabfard/Di-Finder). All possibilities of dinucleotide motifs, consisting of AC, AG, AT, CA, CG, CT, GA, GC, GT, TA, TC, and TG, which were $\geq 6$-repeats were included (Suppl. 2). The written program was based on perfect (pure) STRs. By using the REST API service ${ }^{18}$ from Ensemble 101 (https://asia.ensembl.org) ${ }^{19,20}$, data of three regions of every chromosome, each region spanning 10 Megabase $(\mathrm{Mb})$ of genomic DNA, were accessed in the six species (Fig. 1). In each chromosome, the STR abundances were calculated and compared on a 
chromosome-to-chromosome basis (Table 1, Suppl. 2). Subsequent to collecting the entire data, we also differentiated the STRs based on their length into two classes of 6-20 repeats and >20-repeats, and studied their abundance in the selected species. Finally, the data of the selected regions of the chromosomes in the six species were aggregated and analyzed.

\section{Statistical analysis}

The dinucleotide STR abundance trend in the six selected species was compared across datasets 1,2 , and 3, by correlation coefficient and repeated measurements analysis (Table 2). The Pearson correlation was conducted on the average of STR abundances, consisting of AC, AG, AT, CA, CG, CT, GA, GC, GT, TA, TC, and TG.

\section{Results}

\section{Directional shrinkage of all classes of dinucleotide STRs in primates vs. mouse.}

In three independent analyses, we studied the way dinucleotide STRs were distributed in respect of their abundance across mouse and primates. The observed trend was strikingly decremental in the five primates vs. mouse (Fig. 2). That trend was consistently replicated in datasets 1,2 , and $3(p=0.80)$ (Table 2). The AC class of STRs had the most abundance difference in mouse vs. all primates, followed by TG dinucleotides.

The overall STR counts observed in macaque were an intermediate between mouse and the great apes (Table 2, Fig. 3), which matched the phylogenetic distance of these species.

\section{The >20-repeat dinucleotide STRs were more dramatically affected by shrinkage.}

The $>20$-repeat compartment was the most dramatically affected in respect of shrinkage in primates vs. mouse (Fig. 4). While in mouse, this compartment was on average 10-fold larger than in the five selected primate species, the 6-20 repeat compartment was 2-fold larger.

\section{Discussion}

It is largely unknown whether at the crossroads of speciation, STRs evolved as a result of purifying selection, genetic drift, and/or in a directional manner. Our approach to deciphering the above was simple, and yet robust. We selected species that shared sequential chronological ancestors, and investigated all possible dinucleotide STRs of all possible lengths $\geq 6$-repeats. Our analysis revealed a directional trend in the abundance of dinucleotide STRs during primate speciation.

Dinucleotide STRs are the most abundant class of STRs in the vertebrate genomes, and their global pattern of abundance may shed light on a vastly unknown aspect of evolutionary biology. The replicated trends observed in our three datasets seem to be independent of the genome size of the selected species. Mouse has the highest abundance of STRs in comparison to the five selected primate species, and yet 
its genome is smaller than all those species. This finding is in line with the previous reports of lack of relationship between genome size and abundance of STRs ${ }^{21,22}$.

An alternative hypothesis to the directional shrinkage of the dinucleotide STR compartment in primates is that this compartment has actually expanded in mouse. However, the observation that the overall STR counts in macaque were an intermediate between mouse and the great ape species studied (rodent>Old World monkey>great apes) (Fig. 5), supports the shrinkage hypothesis for at least part of the trend observed in our study. Indeed, while both hypotheses may stand in evolutionary terms, directional shrinkage is a likely explanation based on the species selected. It is possible that there is a mathematical threshold required for the abundance of STRs in various species. This is in line with the hypothesis that STRs function as scaffolds for biological computers ${ }^{23}$. In mouse, the overall number of dinucleotide STRs never declined to the numbers observed in the five primates.

Certain STRs located in the core promoters have been subject to contraction in the process of human and non-human primate evolution ${ }^{24}$. A number of those STRs are identical in formula in primates vs. nonprimates, and the genes linked to those STRs are involved in characteristics that have diverged primates from other mammals, such as craniofacial development, neurogenesis, and spine morphogenesis. It is likely that those STRs functioned as evolutionary switch codes for primate speciation. In line with the

above, structural variants are enriched near genes that diverged in expression across great apes ${ }^{25,26}$. It is speculated that STR variants are more likely than single-nucleotide variants to have epistatic interactions, which can have significant consequences in complex traits, in human as well as model organisms ${ }^{27,28}$. Future studies such as large-scale genome-editing of STRs ${ }^{29}$ in embryonic stem cells and investigation of their differentiation into various cell lineages may be candidate approaches to investigate how the massive and dramatically diverged trend of dinucleotide STRs links to primate speciation and evolution.

\section{Limitations}

Although this study covered over $600 \mathrm{Mb}$ of genomic DNA in each of the six selected species, it can be extended to the entire genome and additional species.

\section{Conclusion}

In conclusion, we propose that massive and directional shrinkage of the dinucleotide STR compartment links to, and probably has a determining impact on primate speciation. This is a prime instance of directional STR trend in multiple speciation.

\section{Declarations}

Acknowledgments 
This research was funded by the University of Social Welfare and Rehabilitation Sciences, Tehran, Iran.

\section{Statement of Ethics}

Not applicable. This was an in silico study, and animals and humans were not directly involved in the study.

\section{Conflict of Interest Statement}

The authors have no conflict of interest to declare.

\section{Funding Sources}

This research was funded by the University of Social Welfare and Rehabilitation Sciences, Tehran, Iran.

\section{Data Availability}

All raw data are available at: https://figshare.com/articles/dataset/Di-Finder_Data/13603274.

\section{References}

1 Mohammadparast, S., Bayat, H., Biglarian, A. \& Ohadi, M. Exceptional expansion and conservation of a CT-repeat complex in the core promoter of PAXBP1 in primates. Am. J. Primatol. 76, 747-756 (2014).

2 Bushehri, A., Barez, M. R. M., Mansouri, S. K., Biglarian, A. \& Ohadi, M. Genome-wide identification of human-and primate-specific core promoter short tandem repeats. Gene 587, 83-90 (2016).

3 Nikkhah, M., Rezazadeh, M., Khorshid, H. R. K., Biglarian, A. \& Ohadi, M. An exceptionally long CArepeat in the core promoter of SCGB2B2 links with the evolution of apes and Old World monkeys. Gene 576, 109-114 (2016).

4 Jakubosky, D. et al. Properties of structural variants and short tandem repeats associated with gene expression and complex traits. Nature Communications 11, 1-15 (2020).

5 Valipour, E. et al. Polymorphic core promoter GA-repeats alter gene expression of the early embryonic developmental genes. Gene 531, 175-179 (2013).

6 Ranathunge, C. et al. Transcribed microsatellite allele lengths are often correlated with gene expression in natural sunflower populations. Mol. Ecol. (2020).

7 Press, M. O., Hall, A. N., Morton, E. A. \& Queitsch, C. Substitutions are boring: Some arguments about parallel mutations and high mutation rates. Trends Genet. 35, 253-264 (2019). 
Fotsing, S. F. et al. The impact of short tandem repeat variation on gene expression. Nat. Genet. 51, 1652-1659 (2019).

$9 \quad$ Arabfard, M., Kavousi, K., Delbari, A. \& Ohadi, M. Link between short tandem repeats and translation initiation site selection. Human genomics 12, 47 (2018).

10 Yap, K. et al. A short tandem repeat-enriched RNA assembles a nuclear compartment to control alternative splicing and promote cell survival. Mol. Cell 72, 525-540 (2018).

11 Srivastava, S., Avvaru, A. K., Sowpati, D. T. \& Mishra, R. K. Patterns of microsatellite distribution across eukaryotic genomes. BMC Genomics 20, 153 (2019).

12 Pavlova, A. et al. Purifying selection and genetic drift shaped Pleistocene evolution of the mitochondrial genome in an endangered Australian freshwater fish. Heredity (Edinb.) 118, 466-476 (2017).

13 Jorde, P. E. et al. Genetically distinct populations of northern shrimp, Pandalus borealis, in the North Atlantic: adaptation to different temperatures as an isolation factor. Mol. Ecol. 24, 1742-1757 (2015).

14 Legrand, D., Chenel, T., Campagne, C., Lachaise, D. \& Cariou, M. L. Inter-island divergence within Drosophila mauritiana, a species of the D. simulans complex: Past history and/or speciation in progress? Mol. Ecol. 20, 2787-2804 (2011).

15 Sun, G. et al. Global genetic variation at nine short tandem repeat loci and implications on forensic genetics. Eur. J. Hum. Genet. 11, 39-49 (2003).

16 Abe, H. \& Gemmell, N. J. Evolutionary footprints of short tandem repeats in avian promoters. Sci. Rep. 6, 1-11 (2016).

17 Lander, E. S. et al. Initial sequencing and analysis of the human genome. (2001).

18 Yates, A. et al. The Ensembl REST API: Ensembl data for any language. Bioinformatics 31, 143145 (2015).

19 Zerbino, D. R. et al. Ensembl 2018. Nucleic Acids Res. 46, D754-D761 (2018).

20 Howe, K. L. et al. Ensembl genomes 2020-enabling non-vertebrate genomic research. Nucleic Acids Res. 48, D689-D695 (2020).

21 Neff, B. D. \& Gross, M. R. Microsatellite evolution in vertebrates: inference from AC dinucleotide repeats. Evolution 55, 1717-1733 (2001).

22 Park, J. Y. et al. Evolutionary constraints over microsatellite abundance in larger mammals as a potential mechanism against carcinogenic burden. Sci. Rep. 6, 1-5 (2016). 
23 Herbert, A. Simple Repeats as Building Blocks for Genetic Computers. Trends Genet. 36(10):739750. (2020).

24 Ohadi, M. et al. Core promoter short tandem repeats as evolutionary switch codes for primate speciation. Am. J. Primatol. 77, 34-43 (2015).

25 Kronenberg, Z. N. et al. High-resolution comparative analysis of great ape genomes. Science $\mathbf{3 6 0}$ (2018).

26. Bilgin Sonay, T. et al. Tandem repeat variation in human and great ape populations and its impact on gene expression divergence. Genome Res. 25, 1591-1599 (2015).

27 Press, M. O. \& Queitsch, C. Variability in a short tandem repeat mediates complex epistatic interactions in Arabidopsis thaliana. Genetics 205, 455-464 (2017).

28 Press, M. O., Carlson, K. D. \& Queitsch, C. The overdue promise of short tandem repeat variation for heritability. Trends Genet. 30, 504-512 (2014).

29 Smith, C. J. et al. Enabling large-scale genome editing at repetitive elements by reducing DNA nicking. Nucleic Acids Res. 48, 5183-5195 (2020).

\section{Tables}

Table 1: Chromosome-by-chromosome study of dinucleotide STR abundance (count) exemplified in Dataset $2^{*}$. 


\section{Human}

\begin{tabular}{l|l|l|l|l|l|l|l|l|l|l|l|l|l|l|l|l|l|l|l|l|} 
Motif & Chr1 & Chr3 & Chr4 & Chr5 & Chr6 & Chr7 & Chr8 & Chr9 & Chr10 & Chr11 & Chr12 & Chr13 & Chr14 & Chr15 & Chr16 & Chr17 & Chr18 & Chr19 & ChrX \\
\hline
\end{tabular}

\begin{tabular}{|l|l|l|l|l|l|l|l|l|l|l|l|l|l|l|l|l|l|l|l|} 
AC & 111 & 133 & 223 & 203 & 208 & 238 & 216 & 207 & 154 & 168 & 150 & 26 & 21 & 5 & 201 & 161 & 92 & 173 & 192 \\
\hline
\end{tabular}

\begin{tabular}{|l|l|l|l|l|l|l|l|l|l|l|l|l|l|l|l|l|l|l|l|} 
AG & 84 & 76 & 84 & 84 & 81 & 62 & 76 & 73 & 61 & 60 & 79 & 4 & 13 & 2 & 85 & 80 & 41 & 115 & 75 \\
\hline
\end{tabular}

\begin{tabular}{|l|l|l|l|l|l|l|l|l|l|l|l|l|l|l|l|l|l|l|l|} 
AT & 84 & 103 & 195 & 188 & 139 & 170 & 197 & 173 & 160 & 122 & 156 & 31 & 17 & 0 & 118 & 135 & 71 & 149 & 136 \\
\hline
\end{tabular}

\begin{tabular}{|l|l|l|l|l|l|l|l|l|l|l|l|l|l|l|l|l|l|l|l|} 
CA & 84 & 75 & 109 & 101 & 104 & 113 & 110 & 92 & 112 & 84 & 74 & 18 & 10 & 3 & 110 & 88 & 48 & 72 & 91 \\
\hline
\end{tabular}

\begin{tabular}{|l|c|c|c|c|c|c|c|c|c|c|c|c|c|c|c|c|c|c|c|} 
CG & 2 & 1 & 1 & 0 & 0 & 0 & 0 & 1 & 0 & 1 & 1 & 0 & 1 & 0 & 0 & 2 & 0 & 4 & 2 \\
\hline
\end{tabular}

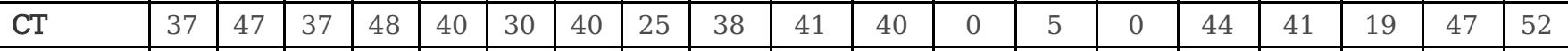

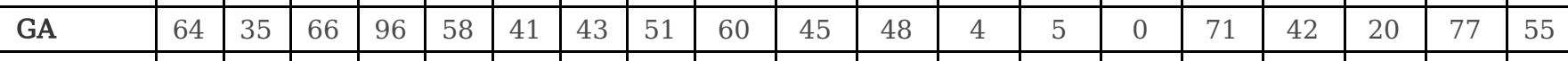

\begin{tabular}{|l|l|l|l|l|l|l|l|l|l|l|l|l|l|l|l|l|l|l|c|} 
GC & 3 & 1 & 0 & 3 & 6 & 2 & 3 & 3 & 1 & 4 & 1 & 0 & 0 & 0 & 2 & 4 & 0 & 4 & 1 \\
\hline
\end{tabular}

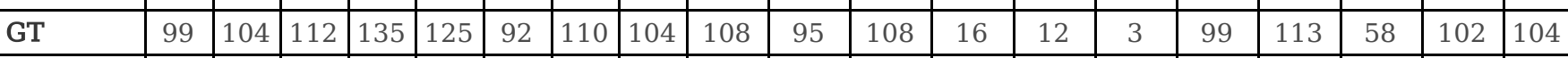

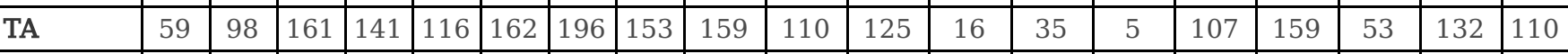

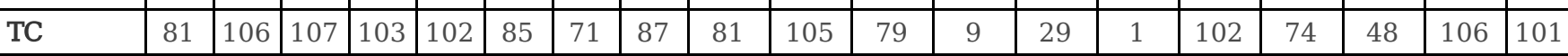

\begin{tabular}{|l|l|l|l|l|l|l|l|l|l|l|l|l|l|l|l|l|l|l|l|} 
TG & 144 & 158 & 180 & 187 & 197 & 175 & 169 & 203 & 161 & 153 & 184 & 26 & 22 & 0 & 184 & 160 & 92 & 148 & 172 \\
\hline
\end{tabular}

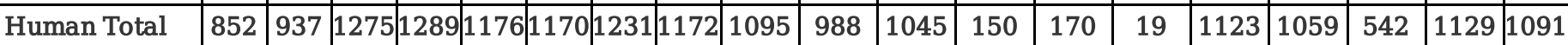

\section{Chimpanzee}

\begin{tabular}{|c|c|c|c|c|c|c|c|c|c|c|c|c|c|c|c|c|c|c|c|}
\hline Motif & Chr1 & Chr3 & Chr4 & Chr5 & Chr6 & Chr7 & Chr8 & Chr9 & Chr10 & Chr11 & Chr12 & Chr13 & Chr14 & Chr15 & Chr16 & Chr17 & Chr18 & Chr19 & ChrX \\
\hline $\mathrm{AC}$ & 115 & 143 & 211 & 203 & 207 & 243 & 234 & 198 & 154 & 153 & 154 & 177 & 185 & 180 & 177 & 154 & 144 & 165 & 12 \\
\hline $\mathrm{AG}$ & 92 & 61 & 98 & 84 & 79 & 49 & 74 & 62 & 72 & 61 & 64 & 70 & 60 & 71 & 91 & 86 & 65 & 114 & \\
\hline $\mathrm{AT}$ & 85 & 98 & 199 & 157 & 140 & 140 & 173 & 184 & 159 & 119 & 157 & 155 & 180 & 148 & 130 & 135 & 160 & 159 & \\
\hline $\mathrm{CA}$ & 86 & 86 & 103 & 141 & 103 & 100 & 103 & 84 & 93 & 88 & 79 & 99 & 89 & 94 & 109 & 99 & 111 & 82 & 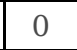 \\
\hline CG & 2 & 1 & 0 & 0 & 3 & 0 & 0 & 4 & 1 & 1 & 0 & 0 & 1 & 2 & 1 & 4 & 2 & 3 & \\
\hline CT & 48 & 46 & 38 & 43 & 36 & 37 & 41 & 25 & 43 & 46 & 41 & 30 & 33 & 32 & 48 & 53 & 30 & 54 & 0 \\
\hline GA & 55 & 40 & 62 & 56 & 57 & 47 & 34 & 43 & 61 & 33 & 45 & 44 & 46 & 42 & 60 & 42 & 42 & 71 & \\
\hline GC & 1 & 2 & 1 & 3 & 6 & 0 & 0 & 2 & 2 & 0 & 2 & 0 & 1 & 1 & 2 & 4 & 2 & 2 & 0 \\
\hline GT & 96 & 101 & 108 & 106 & 131 & 92 & 114 & 95 & 103 & 99 & 96 & 139 & 82 & 105 & 107 & 150 & 103 & 90 & Q \\
\hline TA & 61 & 103 & 146 & 130 & 119 & 139 & 181 & 163 & 126 & 111 & 149 & 124 & 133 & 120 & 110 & 119 & 142 & 132 & 0 \\
\hline $\mathrm{TC}$ & 78 & 91 & 104 & 106 & 103 & 76 & 67 & 73 & 75 & 104 & 89 & 83 & 75 & 80 & 102 & 105 & 81 & 121 & 0 \\
\hline TG & 167 & 163 & 169 & 187 & 191 & 177 & 150 & 171 & 151 & 160 & 184 & 184 & 183 & 182 & 182 & 153 & 161 & 151 & 0 \\
\hline Chimpanzee Total & 886 & 935 & 1239 & 1216 & 1175 & 1100 & 1171 & 1104 & 1040 & 975 & 1060 & 1105 & 1068 & 1057 & 1119 & 1104 & 1043 & 1144 & 12 \\
\hline \multicolumn{20}{|c|}{ Bonobo } \\
\hline Motif & Chr1 & Chr3 & Chr4 & Chr5 & Chr6 & Chr7 & Chr8 & Chr9 & Chr10 & Chr11 & Chr12 & Chr13 & Chr14 & Chr15 & Chr16 & Chr17 & Chr18 & Chr19 & $\mathrm{ChrX}$ \\
\hline $\mathrm{AC}$ & 106 & 142 & 214 & 174 & 205 & 243 & 216 & 194 & 141 & 151 & 143 & 0 & 19 & 0 & 142 & 132 & 132 & 135 & 176 \\
\hline $\mathrm{AG}$ & 67 & 66 & 96 & 79 & 82 & 45 & 70 & 61 & 57 & 62 & 77 & 0 & 14 & 0 & 64 & 73 & 60 & 90 & 70 \\
\hline $\mathrm{AT}$ & 71 & 105 & 183 & 131 & 133 & 155 & 136 & 169 & 145 & 112 & 141 & 0 & 25 & 0 & 77 & 101 & 161 & 113 & 133 \\
\hline $\mathrm{CA}$ & 75 & 83 & 106 & 103 & 108 & 104 & 105 & 89 & 88 & 89 & 81 & 0 & 9 & 0 & 87 & 89 & 111 & 64 & 92 \\
\hline CG & 1 & 0 & 2 & 0 & 3 & 0 & 1 & 1 & 0 & 1 & 1 & 0 & 0 & 0 & 0 & 2 & 2 & 3 & 1 \\
\hline CT & 39 & 47 & 40 & 41 & 43 & 37 & 41 & 27 & 45 & 40 & 36 & 0 & 4 & 0 & 29 & 40 & 36 & 49 & 50 \\
\hline GA & 48 & 30 & 61 & 52 & 49 & 39 & 42 & 36 & 62 & 37 & 42 & 0 & 8 & 0 & 46 & 31 & 43 & 52 & 55 \\
\hline GC & 2 & 1 & 0 & 2 & 4 & 0 & 1 & 1 & 0 & 1 & 0 & 0 & 0 & 0 & 1 & 2 & 1 & 3 & 0 \\
\hline GT & 82 & 92 & 112 & 104 & 130 & 93 & 118 & 94 & 101 & 96 & 103 & 0 & 15 & 0 & 73 & 132 & 88 & 88 & 92 \\
\hline TA & 49 & 95 & 140 & 112 & 104 & 133 & 132 & 141 & 105 & 102 & 143 & 0 & 15 & 0 & 72 & 82 & 114 & 87 & 100 \\
\hline $\mathrm{TC}$ & 69 & 91 & 111 & 108 & 93 & 81 & 65 & 79 & 66 & 78 & 78 & 0 & 11 & 0 & 60 & 90 & 68 & 98 & 101 \\
\hline TG & 131 & 165 & 176 & 188 & 180 & 168 & 162 & 175 & 137 & 153 & 171 & 0 & 22 & 0 & 120 & 108 & 161 & 131 & 154 \\
\hline Bonobo Total & 740 & 917 & 1241 & 1094 & 1134 & 1098 & 1089 & 1067 & 947 & 922 & 1016 & 0 & 142 & 0 & 771 & 882 & 977 & 913 & 1024 \\
\hline \multicolumn{20}{|c|}{ Gorilla } \\
\hline Motif & Chr1 & $\mathrm{Chr} 3$ & Chr4 & Chr5 & Chr6 & Chr7 & Chr8 & Chr9 & Chr10 & Chr11 & Chr12 & Chr13 & Chr14 & Chr15 & Chr16 & Chr17 & Chr18 & Chr19 & ChrX \\
\hline $\mathrm{AC}$ & 102 & 140 & 225 & 195 & 233 & 226 & 234 & 178 & 136 & 143 & 154 & 166 & 187 & 156 & 182 & 173 & 119 & 146 & 176 \\
\hline AG & 86 & 57 & 83 & 88 & 80 & 50 & 69 & 73 & 67 & 56 & 78 & 58 & 65 & 69 & 79 & 80 & 48 & 89 & 78 \\
\hline $\mathrm{AT}$ & 73 & 98 & 185 & 141 & 115 & 158 & 148 & 134 & 139 & 113 & 138 & 146 & 161 & 145 & 106 & 128 & 122 & 123 & 131 \\
\hline $\mathrm{CA}$ & 88 & 80 & 156 & \begin{tabular}{|l|}
90 \\
\end{tabular} & 87 & 100 & 104 & 93 & 87 & 91 & 71 & 89 & 87 & 79 & 95 & 75 & 66 & 73 & 86 \\
\hline
\end{tabular}




\begin{tabular}{|c|c|c|c|c|c|c|c|c|c|c|c|c|c|c|c|c|c|c|c|}
\hline CG & 1 & 1 & 1 & 1 & 1 & 0 & 1 & 1 & 0 & 1 & 2 & 0 & 0 & 1 & 0 & 3 & 1 & 2 & 1 \\
\hline $\mathrm{CT}$ & 38 & 43 & 32 & 50 & 43 & 34 & 42 & 39 & 32 & 36 & 33 & 28 & 41 & 39 & 38 & 41 & 18 & 41 & 35 \\
\hline GA & 58 & 38 & 57 & 62 & 51 & 48 & 45 & 51 & 53 & 40 & 50 & 41 & 38 & 42 & 72 & 49 & 27 & 63 & 50 \\
\hline GC & 2 & 2 & 1 & 0 & 3 & 0 & 1 & 0 & 1 & 0 & 1 & 0 & 0 & 2 & 0 & 2 & 1 & 3 & 1 \\
\hline GT & 84 & 100 & 114 & 120 & 114 & 81 & 136 & 81 & 102 & 100 & 93 & 113 & 92 & 118 & 86 & 125 & 77 & 91 & 93 \\
\hline $\mathrm{TA}$ & 45 & 91 & 148 & 126 & 124 & 133 & 173 & 127 & 136 & 103 & 154 & 115 & 148 & 119 & 96 & 158 & 108 & 120 & 102 \\
\hline TC & 81 & 81 & 106 & 98 & 92 & 77 & 59 & 68 & 69 & 93 & 69 & 68 & 68 & 72 & 93 & 81 & 59 & 105 & 89 \\
\hline TG & 139 & 170 & 179 & 197 & 206 & 174 & 188 & 161 & 152 & 148 & 181 & 175 & 173 & 154 & 161 & 178 & 123 & 144 & 154 \\
\hline Gorilla Total & 797 & 901 & 1287 & 1168 & 1149 & 1081 & 1200 & 1006 & 974 & 924 & 1024 & 999 & 1060 & 996 & 1008 & 1093 & 769 & 1000 & 996 \\
\hline \multicolumn{20}{|c|}{ Macaque } \\
\hline Motif & Chr1 & Chr3 & Chr4 & Chr5 & Chr6 & Chr7 & Chr8 & Chr9t & Chr10 & Chr11 & Chr12 & Chr13 & Chr14 & Chr15 & Chr16 & Chr17 & Chr18 & Chr19 & ChrX \\
\hline $\mathrm{AC}$ & 228 & 194 & 228 & 313 & \begin{tabular}{|l|}
216 \\
\end{tabular} & 185 & 240 & 185 & 207 & 187 & 242 & 193 & 179 & 158 & 176 & 195 & 282 & 174 & 190 \\
\hline AG & 104 & 115 & \begin{tabular}{|l|}
68 \\
\end{tabular} & 93 & \begin{tabular}{|l|}
98 \\
\end{tabular} & 64 & 73 & 78 & 93 & 81 & 89 & 87 & 66 & 75 & 89 & 100 & 86 & 104 & 86 \\
\hline $\mathrm{AT}$ & 121 & 144 & 140 & \begin{tabular}{|l|}
162 \\
\end{tabular} & 170 & 127 & 144 & 137 & 118 & 167 & 125 & 113 & 128 & 107 & 109 & 122 & 188 & 132 & 126 \\
\hline $\mathrm{CA}$ & 122 & 125 & \begin{tabular}{|l|}
86 \\
\end{tabular} & 112 & \begin{tabular}{|l|l|}
104 \\
\end{tabular} & 105 & \begin{tabular}{|l|}
88 \\
\end{tabular} & 98 & 122 & 80 & 113 & 71 & 77 & 99 & 115 & 112 & 125 & 97 & 97 \\
\hline CG & \begin{tabular}{|l|}
1 \\
\end{tabular} & 2 & 1 & \begin{tabular}{|l|}
0 \\
\end{tabular} & \begin{tabular}{|l|}
2 \\
\end{tabular} & 2 & 0 & 2 & 2 & 3 & 0 & 0 & 1 & 4 & 3 & 0 & 1 & 0 & 2 \\
\hline CT & 46 & 53 & 42 & 67 & 52 & 51 & 42 & 47 & 57 & 30 & 51 & 35 & 42 & 39 & 40 & 34 & 47 & 96 & 54 \\
\hline GA & 51 & 59 & 50 & 70 & 71 & 60 & 56 & 59 & 61 & 38 & 47 & 44 & 41 & 44 & 49 & 40 & 60 & 74 & 101 \\
\hline GC & 4 & 0 & \begin{tabular}{|l|}
2 \\
\end{tabular} & \begin{tabular}{|l|}
4 \\
\end{tabular} & \begin{tabular}{|l|}
2 \\
\end{tabular} & \begin{tabular}{|l|}
3 \\
\end{tabular} & 1 & 3 & 6 & 0 & 1 & 2 & 4 & 4 & 4 & 1 & 1 & 2 & 2 \\
\hline GT & 92 & 148 & 119 & 155 & 112 & 102 & \begin{tabular}{|l|}
96 \\
\end{tabular} & 125 & 119 & 82 & 109 & 89 & 94 & 90 & 108 & 108 & 114 & 92 & 105 \\
\hline TA & 93 & 101 & 107 & 126 & 124 & 107 & \begin{tabular}{|l|}
111 \\
\end{tabular} & 103 & 89 & 105 & 89 & 95 & 82 & 77 & 93 & 101 & 154 & 84 & 96 \\
\hline $\mathrm{TC}$ & 121 & 124 & \begin{tabular}{|l|}
79 \\
\end{tabular} & \begin{tabular}{|l|}
133 \\
\end{tabular} & \begin{tabular}{|l|l|}
110 \\
\end{tabular} & 94 & \begin{tabular}{l|}
69 \\
\end{tabular} & 107 & 77 & 66 & 95 & 84 & 88 & 72 & 89 & 67 & 97 & 175 & 98 \\
\hline TG & 195 & \begin{tabular}{|l|l|}
199 \\
\end{tabular} & 184 & \begin{tabular}{|l|}
204 \\
\end{tabular} & \begin{tabular}{|l|l|}
222 \\
\end{tabular} & 184 & \begin{tabular}{|l|}
169 \\
\end{tabular} & 237 & 172 & 206 & 205 & 177 & 180 & 140 & 177 & 173 & 264 & 146 & 242 \\
\hline Macaque Total & 1178 & 1264 & 1106 & 1439 & 1283 & 1084 & 1089 & 1181 & 1123 & 1045 & 1166 & 990 & 982 & 909 & 1052 & 1053 & 1419 & 1176 & 1199 \\
\hline \multicolumn{20}{|c|}{ Mouse } \\
\hline Core & Chr1 & Chr3 & Chr4 & Chr5 & Chr6 & Chr7 & Chr8 & Chr9t & Chr10 & Chr11 & Chr12 & Chr13 & Chr14 & Chr15 & Chr16 & Chr17 & Chr18 & Chr19 & ChrX \\
\hline $\mathrm{AC}$ & 485 & 353 & 455 & \begin{tabular}{|l|}
468 \\
\end{tabular} & \begin{tabular}{|l|l|}
525 \\
\end{tabular} & 297 & 612 & 449 & 602 & 447 & 583 & 542 & 485 & 575 & 473 & 505 & 589 & 516 & 470 \\
\hline AG & 214 & \begin{tabular}{|l|}
189 \\
\end{tabular} & 216 & 172 & \begin{tabular}{|l|}
193 \\
\end{tabular} & 200 & \begin{tabular}{|l|}
186 \\
\end{tabular} & 188 & 239 & 200 & 214 & 185 & 179 & 226 & 174 & 165 & 178 & 199 & 189 \\
\hline $\mathrm{AT}$ & 163 & 247 & 201 & 260 & \begin{tabular}{|l|}
241 \\
\end{tabular} & 158 & 144 & 188 & 202 & 212 & 143 & 176 & 143 & 329 & 112 & 163 & 182 & 190 & 183 \\
\hline $\mathrm{CA}$ & 268 & \begin{tabular}{|l|l|}
199 \\
\end{tabular} & 249 & 245 & \begin{tabular}{|l|}
292 \\
\end{tabular} & 233 & \begin{tabular}{|l|}
404 \\
\end{tabular} & 309 & 295 & 242 & 324 & 262 & 311 & 266 & 314 & 262 & 329 & 278 & 272 \\
\hline CG & 8 & 4 & 10 & 3 & 4 & 3 & 8 & 2 & 4 & 4 & 2 & 3 & 5 & 1 & 9 & 5 & 6 & 7 & 6 \\
\hline CT & 139 & 96 & 138 & 115 & 149 & 136 & 128 & 134 & 162 & 118 & 198 & 137 & 133 & 130 & 124 & 130 & 120 & 138 & 149 \\
\hline GA & 165 & 154 & 160 & 153 & 180 & 170 & 187 & 158 & 225 & 155 & 226 & 183 & 148 & 185 & 162 & 153 & 186 & 202 & 203 \\
\hline GC & \begin{tabular}{|l|}
4 \\
\end{tabular} & 3 & \begin{tabular}{|l|}
10 \\
\end{tabular} & 5 & \begin{tabular}{|l|}
4 \\
\end{tabular} & \begin{tabular}{|l|}
8 \\
\end{tabular} & \begin{tabular}{|l|}
6 \\
\end{tabular} & \begin{tabular}{l|l|}
8 \\
\end{tabular} & 9 & 1 & 1 & 9 & 8 & 4 & 5 & 4 & 8 & 3 & 8 \\
\hline GT & 294 & 227 & 291 & 214 & 249 & 198 & 448 & 311 & 375 & 259 & 359 & 333 & 324 & 317 & 329 & 317 & 328 & 361 & 272 \\
\hline TA & 141 & 172 & 180 & 190 & 220 & 90 & 118 & 181 & 176 & 176 & 138 & 165 & 108 & 245 & 92 & 128 & 185 & 186 & 156 \\
\hline $\mathrm{TC}$ & 251 & 188 & 234 & 236 & \begin{tabular}{|l|}
276 \\
\end{tabular} & 198 & 257 & 245 & 264 & 246 & 319 & 286 & 250 & 266 & 200 & 230 & 256 & 295 & 278 \\
\hline TG & 433 & 365 & \begin{tabular}{|l|}
462 \\
\end{tabular} & 438 & \begin{tabular}{|l|}
467 \\
\end{tabular} & 323 & 551 & 411 & 620 & 455 & 647 & 494 & 434 & 493 & 463 & 460 & 523 & 518 & 438 \\
\hline Mouse Total & 2565 & 2197 & 2606 & 2499 & 2800 & 2014 & 3049 & 2584 & 3173 & 2515 & 3154 & 2775 & 2528 & 3037 & 2457 & 2522 & 2890 & 2893 & 2624 \\
\hline
\end{tabular}

*The entire datasets are provided as Suppl. 2 .

Table 2. Repeated measurements analysis of datasets 1, 2, and 3. 


\begin{tabular}{|l|c|r|r|r|r|c|}
\hline & Human & Chimpanzee & Bonobo & Gorilla & Macaque & Mouse \\
\hline Dataset 1 & 1515.92 & 1456.92 & 1255.25 & 1567.25 & 2025.50 & 3007.83 \\
Dataset 2 & 1459.42 & 1629.42 & 1331.17 & 1619.33 & 1811.50 & 4240.17 \\
Dataset 3 & 1751.67 & 1691.92 & 1581.42 & 1636.50 & 1780.17 & 4545.50 \\
\hline p-value & \multicolumn{6}{|c|}{0.801} \\
\hline
\end{tabular}

Correlation between dataset 1 and 2: 0.960418, $\mathrm{p}=0.002319$

Correlation between datasets 1 and 3: 0.935812, $\mathrm{p}=0.006048$

Correlation between datasets 2 and 3: 0.992976, $\mathrm{p}=0.000074$

\section{Figures}

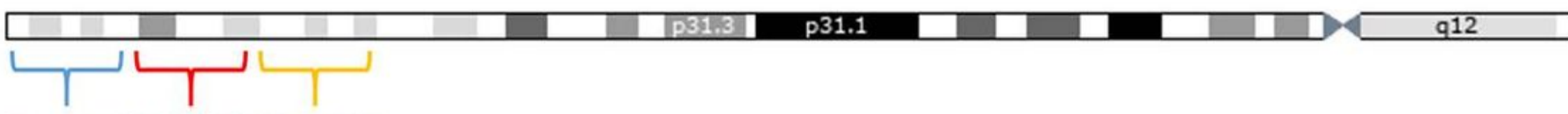

Dataset 1 Dataset 2 Dataset 3

\section{Figure 1}

Schematic representation of data collection of the dinucleotide STR compartment. All chromosomes were screened across the six selected species in three datasets 1,2 , and 3 . Only one chromosome is depicted as an example. 

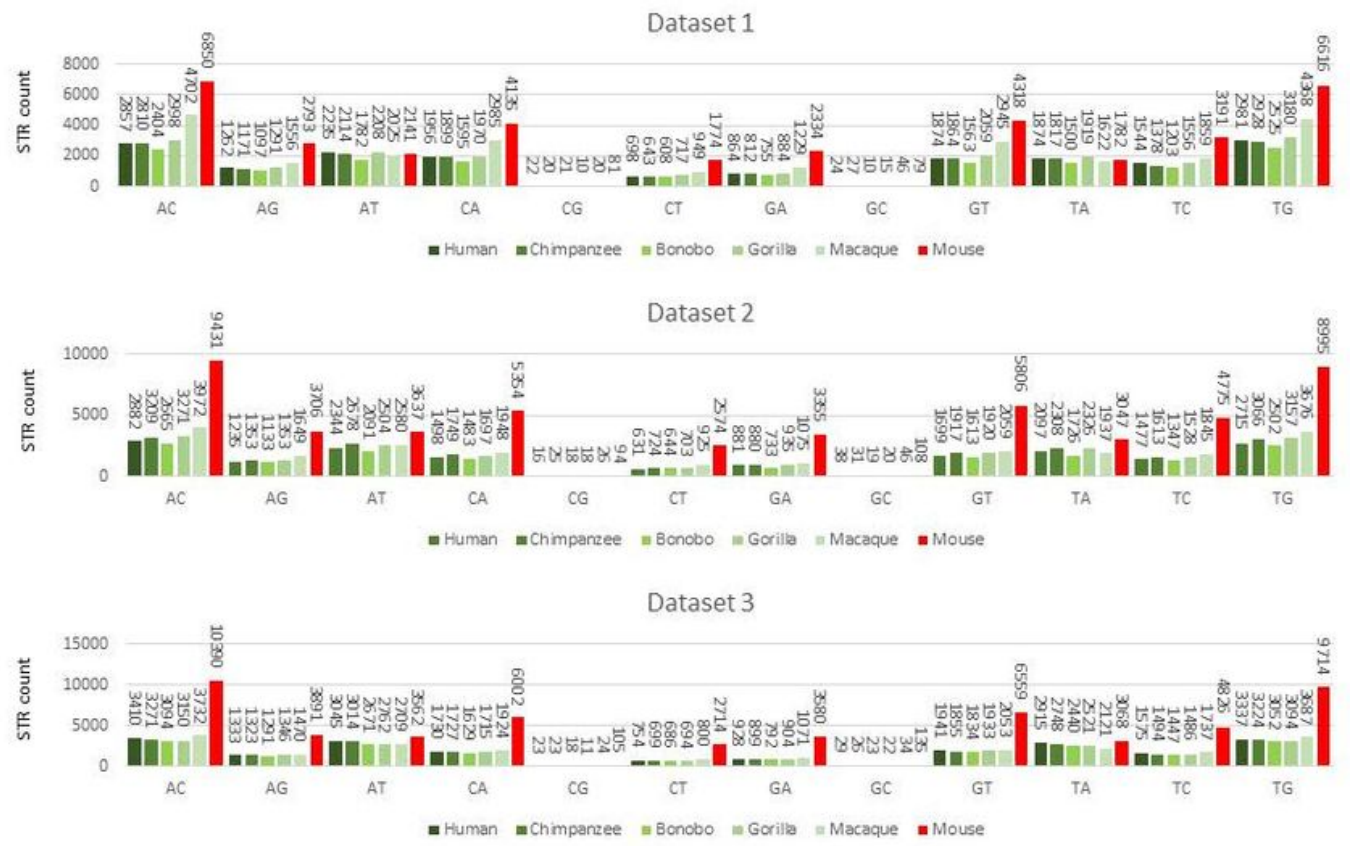

\section{Figure 2}

Abundance of the various dinucleotide STR classes across the six selected species. All classes of dinucleotide STRs were consistently more abundant in mouse vs. all five primates studied.

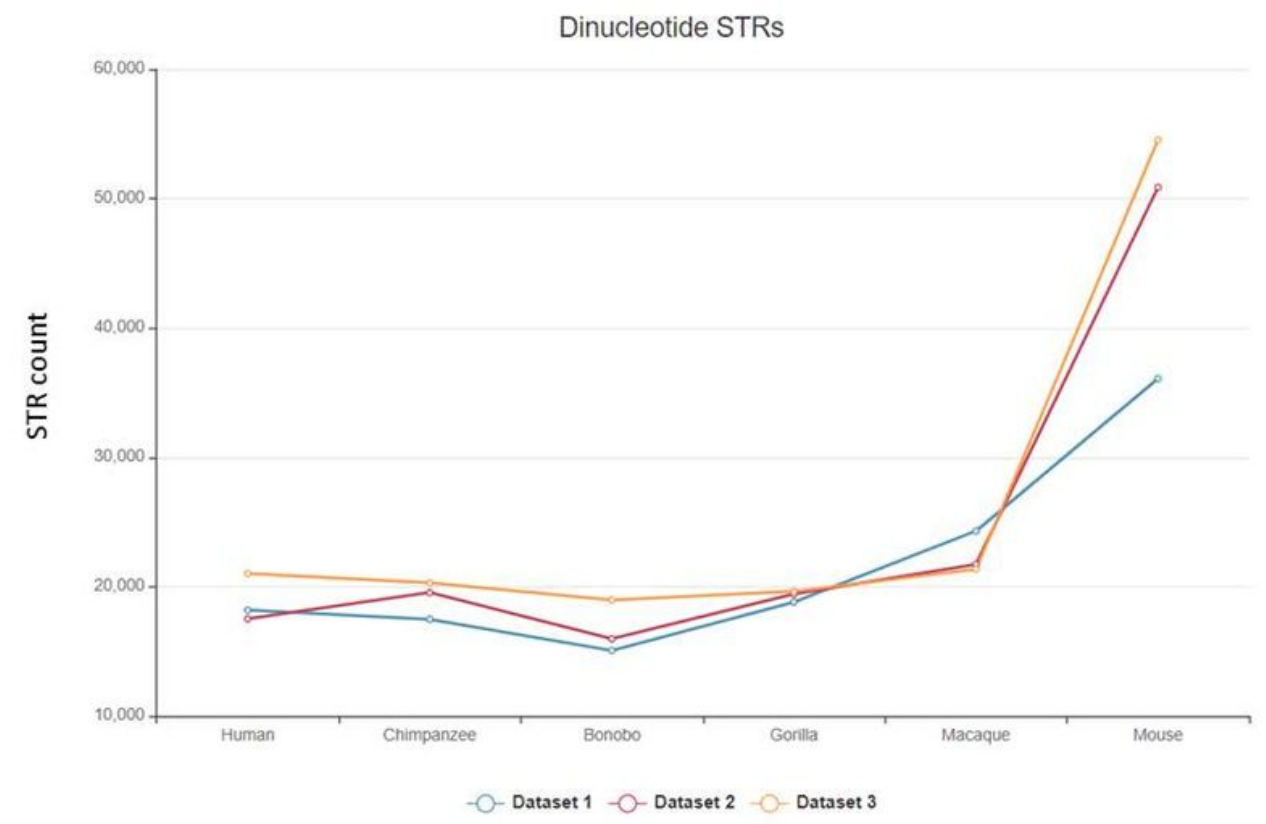


Figure 3

Massive shrinkage of the dinucleotide STR compartment in primates, replicated in datasets 1, 2, and 3.

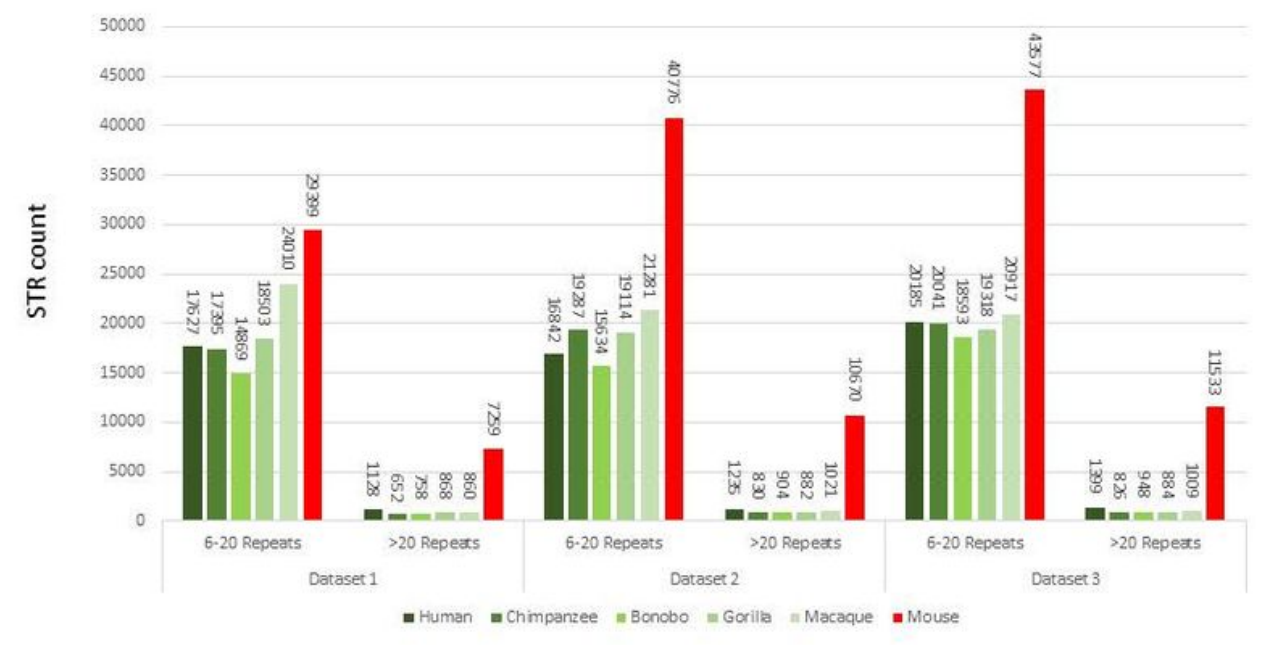

\section{Figure 4}

Abundance of dinucleotide STRs across mouse and primates based on length. We differentiated dinucleotide STRs based on length in two classes of 6-20-repeats and $>20$-repeats. While both classes were shrunk in primates vs. mouse, the $>20$-repeat compartment was more dramatically affected. 


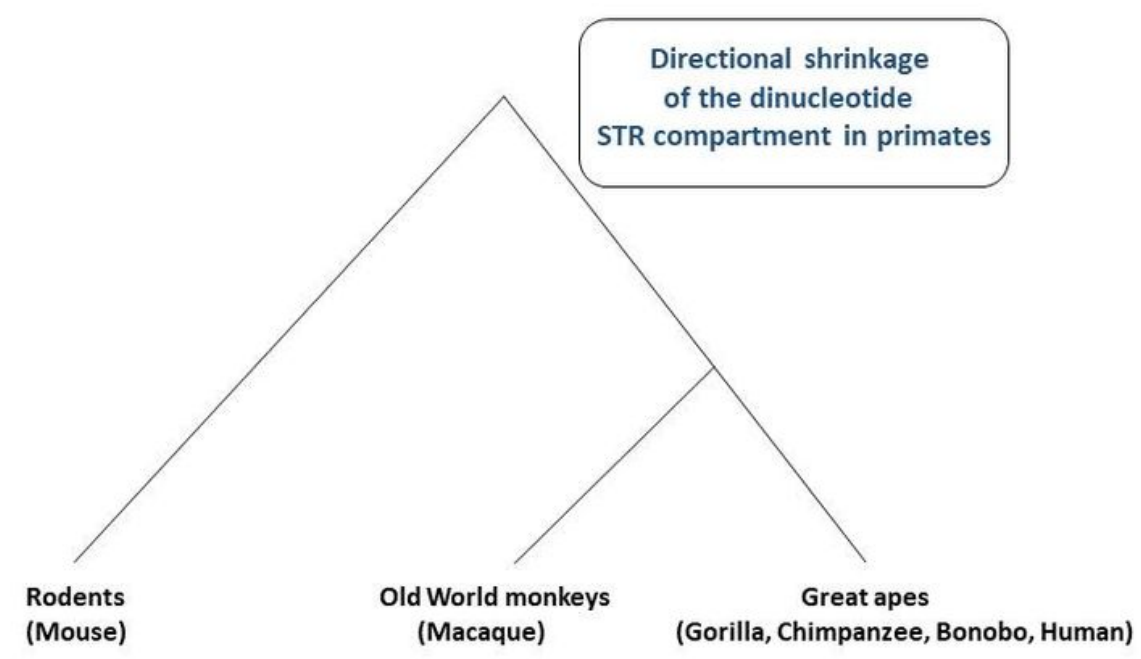

Figure 5

Proposed trend of dinucleotide STRs in primate speciation.

\section{Supplementary Files}

This is a list of supplementary files associated with this preprint. Click to download.

- Suppl.1.DiFinder.pdf

- Suppl.2..pdf 